\title{
AN ESTIMATE ON THE EIGENVALUES IN BIFURCATION FOR GRADIENT MAPPINGS
}

\author{
by RAFFAELE CHIAPPINELLI
}

(Received 21 December, 1995)

1. Introduction. Let $H$ be a real Hilbert space and let $A: H \rightarrow H$ be a nonlinear operator such that $A(0)=0$. We consider the eigenvalue problem

$$
A(x)=\lambda x \quad(\lambda \in \mathbb{R}, x \neq 0) .
$$

Recall that $\lambda_{0} \in \mathbb{R}$ is said to be a bifurcation point for (1.1) if every neighbourhood of $\left(\lambda_{0}, 0\right)$ in $\mathbb{R} \times H$ contains solutions of (1.1).

In the variational case-namely, when $A$ is the gradient of a functional $\phi$ on $H$-a classical theorem of Krasnosel'skii guarantees, under suitable assumptions on $A$ and $\phi$, the existence of infinitely many bifurcation points for (1). To that purpose, let us recall that a functional $\phi: H \rightarrow \mathbb{R}$ is said to be weakly continuous if it maps weakly convergent sequences of $H$ into convergent sequences; while an operator $A: H \rightarrow H$ is said to be completely continuous if it is continuous and compact (i.e., it maps bounded sets into sets having compact closure). Krasnosels'kii result ([6], Theorem 6.2.2) can then be stated as follows.

THEOREM 0. Assume that $\phi: H \rightarrow \mathbb{B}$ is weakly continuous and uniformly differentiable in a neighbourhood of 0 , and assume that $A=\operatorname{grad} \phi: H \rightarrow H$ is completely continuous. Then, if $A$ is differentiable at 0 , every eigenvalue $\lambda_{0} \neq 0$ of the derivative $A^{\prime}(0)$ is $a$ bifurcation point for (1).

More precisely, for any sufficiently small $r>0$ there exists $\lambda_{r} \in \mathbb{R}, x_{r} \in H$ with $\left\|x_{r}\right\|=r$ such that $A\left(x_{r}\right)=\lambda_{r} x_{r}$, and furthermore

$$
\lambda_{r} \rightarrow \lambda_{0} \quad(r \rightarrow 0) .
$$

Recall that under the above assumptions $A^{\prime}(0)$ is a (linear) compact self-adjoint operator, so that "generically" it has infinitely many nonzero eigenvalues. We refer to [6] for the concept of uniform differentiability, which is however easy to imagine.

Though the proof of the above Theorem is far from immediate, its basic lines run as follows. Finding an $x \in H$ with $\|x\|=r>0$ which solves (1.1), i.e.

$$
\operatorname{grad} \phi(x)=\lambda x
$$

for some $\lambda \in \mathbb{R}$, is equivalent to finding a constrained critical point of $\phi$ on the sphere $S_{r}=\{x \in H:\|x\|=r\}$. These critical points are obtained in [6] by a "minimax" processover a certain class of compact subsets of $S_{r}$-that we shall shortly recall later. Formula (1.2) for the eigenvalues is then a consequence of the differentiability of $A$ in 0 . Our point in this note is that, if the differentiability assumption is strengthened, then more precise information on the behaviour of $\lambda_{r}$ as $r \rightarrow 0$ can be gained. To be clearer, consider Taylor's formula for $A$ at 0 :

$$
A(x)=T x+R(x)
$$

Glasgow Math. J. 39 (1997) 211--216. 
where $T=A^{\prime}(0)$ and the remainder term $R(x)$ is $o(\|x\|)$ as $\|x\| \rightarrow 0$. Assuming $R(x)=O\left(\|x\|^{p}\right)$ as $\|x\| \rightarrow 0$ with $p>1$, we prove that rather than (1.2) one gets

$$
\lambda_{r}=\lambda_{0}+O\left(r^{p-1}\right) \quad(r \rightarrow 0) .
$$

We also show that such condition appears naturally in the applications of bifurcation theory to nonlinear elliptic eigenvalue problems like

$$
\begin{cases}-\Delta u=\mu(u+f(x, u)), & x \in \Omega \\ u(x)=0, & x \in \partial \Omega\end{cases}
$$

where $\Omega$ is an open bounded subset of $\mathbb{R}^{n}$ and $f=f(x, s)$ is a function on $\Omega \times \mathbb{R}$ satisfying the usual growth assumptions in $s$ : see e.g. [4], [8].

Remark. In the last decades, Theorem 0 has been established in greater generality. Essentially, it is sufficient to consider any isolated eigenvalue of finite multiplicity of the derivative $A^{\prime}(0)$ without requiring any compactness: see e.g. [10]. We believe that our sharpening of the conclusion also holds in this generality.

2. Asymptotic behaviour of the eigenvalues. In order to prove our result, we need recall the main points of the method followed in [6]. Let therefore $\left(\lambda_{i}\right)$ be the nonzero eigenvalues of the compact self-adjoint operator $T=A^{\prime}(0)$, repeated according to multiplicities, and let $\left(e_{i}\right)$ be the corresponding orthonormal eigenvectors; we then have

$$
(T x, x)=\sum_{i=1}^{\infty} \lambda_{i}\left|\left(x, e_{i}\right)\right|^{2} \quad \forall x \in H .
$$

Let $\lambda_{0}$ be one of the eigenvalues $\lambda_{i}$; suppose, to fix the ideas, $\lambda_{0}>0$. Denote by $H_{0}$ the eigenspace associated with $\lambda_{0}$ and by $H_{1}$ the sum of the eigenspaces associated to the eigenvalues $\lambda_{i} \geq \lambda_{0}$, i.e.

$$
H_{1}=\operatorname{span}\left\{e_{i}: T e_{i}=\lambda_{i} e_{i}, \lambda_{i} \geq \lambda_{0}\right\} .
$$

We thus have $(T x, x)=\lambda_{0}\|x\|^{2}$ if $x \in H_{0}$ and $(T x, x) \geq \lambda_{0}\|x\|^{2}$ if $x \in H_{1}$. Next set $R=\left\{x \in H: P_{1} x \neq 0\right\}$, with $P_{1}$ the orthogonal projection on $H$ onto $H_{1}$, and

$$
M_{r}=\left\{F \subset S_{r} \cap R ; F \text { compact, noncontractible in } R\right\} .
$$

(Recall that a subset $F$ of a topological space $R$ is said to be contractible (in $R$ ) to the point $x_{0} \in R$ if there exists a continuous map $U:[0,1] \times F \rightarrow R$ such that $U(0, x)=x$ and $U(1, x)=x_{0}$ for any $\left.x \in F\right)$. We finally set

$$
c_{r}=\sup _{F \in M_{r}} \min \phi(x)
$$

and note this is a good definition since $M_{r}$ is nonempty, as we shall see later. Now, Krasnosels'skii proves that, for $r>0$ less than some $r_{0}, c_{r}$ is a critical value of $\phi$ on $S_{r}$ : namely, there exist $x_{r} \in S_{r}, \lambda_{r} \in \mathbb{R}$ so that

$$
\phi\left(x_{r}\right)=c_{r} \text { and } A\left(x_{r}\right)=\lambda_{r} x_{r} .
$$

Moreover, the condition $R(x)=A(x)-T x=o(\|x\|)$-expressing the differentiability of $A$ at 0 -is used to show that $\lambda_{r} \rightarrow \lambda_{0}$, which proves the bifurcation from $\lambda_{0}$. We have the following improvement to this result:

THEOREM 1. Under the same assumptions as in Theorem 0, suppose further that the 
remainder term $R$ satisfies $R(x)=O\left(\|x\|^{p}\right)$ for $x \rightarrow 0$ with $p>1$. Then the eigenvalues $\lambda_{r}$ satisfy

$$
\lambda_{r}=\lambda_{0}+O\left(r^{p-1}\right) \quad(r \rightarrow 0) .
$$

Proof. Let $k>0, r_{0}>0, p>1$ be such that

$$
\|R(x)\| \leq k\|x\|^{p} \quad\left(\|x\|<r_{0}\right) .
$$

Recall (e.g. [2], [9]) that $\phi(x)=\int_{0}^{1}(A(t x), x) d t$ and so

$$
\phi(x)=\frac{1}{2}(T x, x)+\int_{0}^{1}(R(t x), x) d t .
$$

From (2.6) it then follows that

$$
\left|\phi(x)-\frac{1}{2}(T x, x)\right| \leq\|x\| \int_{0}^{1}\|R(t x)\| d t \leq \frac{k}{p+1}\|x\|^{p+1} \quad\left(\|x\|<r_{0}\right) .
$$

Since $p>1$, one thus obtains

$$
\frac{1}{2}(T x, x)-\frac{k}{2} r^{p+1} \leq \phi(x) \leq \frac{1}{2}(T x, x)+\frac{k}{2} r^{p+1}
$$

for any $x:\|x\| \leq r<r_{0}$. We can now estimate $c_{r}$ and prove that

$$
\left|c_{r}-\frac{1}{2} \lambda_{0} r^{2}\right| \leq \frac{k}{2} r^{p+1} \quad\left(r<r_{0}\right) .
$$

To this end, we need two basic properties of the family $M_{r}[6]$ :

(i) $S_{r}^{1} \in M_{r}$, where $S_{r}^{1}=S_{r} \cap H_{1}$,

(ii) for any $F \in M_{r}$, there exists $z \in F$ such that $P_{1} z \in H_{0}$.

From (i) it follows that

$$
c_{r}=\sup _{F \in M_{r}} \min _{x \in F} \phi(x) \geq \min _{x \in S_{r}^{\prime}} \phi(x) .
$$
$\left.H_{1}\right)$

On the other hand if $x \in S_{r}^{1}, r<r_{0}$, (2.7) yields (on recalling that $(T x, x) \geq \lambda_{0}\|x\|^{2}$ on

$$
\phi(x) \geq \frac{1}{2} \lambda_{0} r^{2}-\frac{k}{2} r^{p+1},
$$

which gives one half of the estimate (8). To prove that

$$
c_{r} \leq \frac{1}{2} \lambda_{0} r^{2}+\frac{k}{2} r^{p+1}
$$

we first let $H_{2}$ denote the orthogonal subspace to $H_{1}$; thus, writing $x=x_{1}+x_{2}$ with $x_{1}=P_{1} x \in H_{1}, x_{2} \in H_{2}$, we have (by virtue of (2.1)) $\left(T x_{2}, x_{2}\right) \leq \lambda_{0}\left\|x_{2}\right\|^{2}$.

Let now $F \in M_{r}$; by (ii), there exists $z \in F$ such that $z_{1}=P_{1} z \in H_{0}$ and so

$$
(T z, z)=\left(T z_{1}, z_{1}\right)+\left(T z_{2}, z_{2}\right) \leq \lambda_{0}\left(\left\|z_{1}\right\|^{2}+\left\|z_{2}\right\|^{2}\right)=\lambda_{0}\|z\|^{2} .
$$

On using this inequality in (2.7), we obtain

$$
\min _{x \in F} \phi(x) \leq \gamma(z) \leq \frac{1}{2} \lambda_{0} r^{2}+\frac{k}{2} r^{p+1}
$$

and since this is true for any $F \in M_{r}$, the result follows. 
We now go on to estimate $\lambda_{r}$, the eigenvalue corresponding to $x_{r} \in S_{r}$ with $\phi\left(x_{r}\right)=c_{r}$. One has $\lambda_{r} r^{2}=\left(A\left(x_{r}\right), x_{r}\right)$ and so

$$
\begin{aligned}
\lambda_{r}-\lambda_{0} & =\frac{1}{r^{2}}\left[\left(A\left(x_{r}\right), x_{r}\right)-\lambda_{0}\left(x_{r}, x_{r}\right)\right] \\
& =\frac{1}{r^{2}}\left(A\left(x_{r}\right)-T x_{r}+T x_{r}-\lambda_{0} x_{r}, x_{r}\right)
\end{aligned}
$$

whence, by virtue of (2.6),

$$
\begin{aligned}
\left|\lambda_{r}-\lambda_{0}\right| & \leq \frac{1}{r^{2}}\left\|A\left(x_{r}\right)-T x_{r}\right\|\left\|x_{r}\right\|+\frac{2}{r^{2}}\left|\frac{\left(T x_{r}, x_{r}\right)}{2}-\lambda_{0} \frac{r^{2}}{2}\right| \\
& \leq \frac{1}{r^{2}} k r^{p+1}+\frac{2}{r^{2}}\left|\frac{\left(T x_{r}, x_{r}\right)}{2}-\phi\left(x_{r}\right)+c_{r}-\lambda_{0} \frac{r^{2}}{2}\right|
\end{aligned}
$$

Finally, using (2.7) and the estimate (2:8) for $c_{r}$ we have

$$
\left|\lambda_{r}-\lambda_{0}\right| \leq k r^{p-1}+2 k r^{p-1}=3 k r^{p-1} \quad\left(r<r_{0}\right)
$$

which concludes the proof of Theorem 1 .

3. An application. Let $\Omega$ be an open bounded subset of $\mathbb{R}^{n}$. Consider the nonlinear eigenvalue problem

$$
\begin{cases}-\Delta u=\mu(u+f(x, u)), & x \in \Omega \\ u(x)=0, & x \in \partial \Omega\end{cases}
$$

where $f=f(x, t): \Omega \times \mathbb{R} \rightarrow \mathbb{R}$ is a Caratheodory function (i.e. continuous in $t$ for a.a. $x \in \Omega$ and measurable in $x$ for any $t \in \mathbb{R}$ ) satisfying the growth condition

$$
\left.|f(x, t)| \leq a|t|^{p} \quad \text { (a.a. } x \in \Omega, t \in \mathbb{R}\right)
$$

for some $a \geq 0$ and some $p: 1<p<\frac{n+2}{n-2}$ if $n>2,1<p<\infty$ if $n \leq 2$.

We consider the real Sobolev space $H=W_{0}^{1,2}(\Omega)$, which is a Hilbert space under the scalar product $(u, v)=\int_{\Omega} \nabla u . \nabla v$, and recall that a weak solution of $(P)$ is a function $u \in H$ such that

$$
\int_{\Omega} \nabla u . \nabla v=\mu\left(\int_{\Omega} u v+\int_{\Omega} f(x, u) v\right) \quad \forall v \in H .
$$

If $u \neq 0$ we say that $u$ is an eigenfunction of (3.1) associated to the eigenvalue $\mu$; note that by (3.2) $f(x, 0)=0$ for a.a. $x \in \Omega$, so that $u=0$ is a solution for any $\mu \in \mathbb{R}$.

Let $0<\mu_{1}<\mu_{2} \leq \ldots$ denote the eigenvalues of the linear problem

$$
\begin{cases}-\Delta u=\mu u, & x \in \Omega \\ u(x)=0, & x \in \partial \Omega\end{cases}
$$

THEOREM 2. Let $\mu_{0}$ be an eigenvalue of (3.4). For any sufficiently small $r>0$, there exists an eigenvalue $\mu_{r}$ of (3.1) with corresponding eigenfunction $u_{r} \in H$ such that $\|u\|=r$. We have $\mu_{r} \rightarrow \mu_{0}$ as $r \rightarrow 0$ (i.e., $\mu_{0}$ is a bifurcation point for (3.1)), and more precisely

$$
\mu_{r}=\mu_{0}+O\left(r^{p-1}\right) \text { as } r \rightarrow 0 \text {. }
$$

Proof. We shall limit ourselves to sketch the relevant points; for a detailed discussion of semilinear elliptic problems like (3.1), standard references are e.g. [4], [8]. 
Define operators $T, R$ in $H$ by the rules

$$
(T u, v)=\int_{\Omega} u v, \quad(R(u), v)=\int_{\Omega} f(x, u) v
$$

for $u, v \in H$; then (3.3) becomes

$$
(u, v)=\mu[(T u, v)+(R(u), v)] \quad \forall v \in H
$$

so that $u$ is a weak solution of (3.1) if and only if

$$
A(u):=T u+R(u)=\lambda u
$$

where $\lambda=\mu^{-1}(\mu \neq 0)$. $A$ is the gradient of the functional

$$
\phi(u)=\frac{1}{2} \int_{\Omega} u^{2}+\int_{\Omega} F(x, u)
$$

where $F(x, t)=\int_{0}^{t} f(x, s) d s$. We now check that

$$
\|R(u)\| \leq c\|u\|^{p}
$$

for some $c>0$ and all $u \in H(\|$.$\| is the norm in H$ ). Indeed, using (3.2) and Holder's inequality with exponents $p+1,(p+1) / p$ one has

$$
\begin{aligned}
\left|\int_{\Omega} f(x, u) v\right| \leq a \int_{\Omega}|u|^{p}|v| & \leq a\left(\int_{\Omega}|u|^{p+1}\right)^{p /(p+1)}\left(\int_{\Omega}|v|^{p+1}\right)^{1 /(p+1)} \\
& =a\|u\|_{L^{p+1}(\Omega)}^{p}\|v\|_{L^{p+1}(\Omega)} \leq c\|u\|^{p}\|v\|
\end{aligned}
$$

where the last inequality is a consequence of the Sobolev embedding $H \subset L^{p+1}(\Omega)$; (3.6) now follows readily from the definition of $R$. The compactness of this embeddingtogether with well-known properties of the Nemitskii operator in $L^{p}$ spaces $([4]$, [8])-also shows that $R$ is not only completely continuous but in fact strongly continuous, namely it maps weakly convergent sequences into strongly convergent ones. This in turn implies that $\phi$ is weakly continuous and uniformly differentiable on each bounded subset of $H$ (see e.g. [1], Lemma 3.2 and [9], Theorem 4.2).

We thus see that all conditions of Theorem 1 are satisfied and-since the eigenvalues of $T$ are clearly the reciprocals of the eigenvalues $\mu_{n}$ of (3.4)-we deduce that if $\mu_{0}$ is one such eigenvalue, then for small $r>0$ there exist a solution pair $\left(\lambda_{r}, u_{r}\right) \in \mathbb{R} \times H$ of (3.1) with $\left\|u_{r}\right\|=r$ while $\lambda_{r}=\mu_{0}^{-1}+O\left(r^{p-1}\right)$ as $r \rightarrow 0$. This yields immediately the corresponding formula (3.5) for $\mu_{r}=\lambda_{r}^{-1}$.

REMARK. Recall (see e.g. [7], Remark 3.3) that the Nemitskii operator induced by a Caratheodory function maps $L^{p+1}(\Omega)$ into $L^{q}(\Omega)(q=(p+1) / p)$ if and only if there exist $a>0$ and $b \in L^{q}(\Omega)$ such that

$$
|f(x, t)| \leq a|t|^{p}+b(x) \quad(\text { a.a. } x \in \Omega, t \in \mathbb{R}) .
$$

In the applications to eigenvalue problems, one takes $b=0$ in (3.6), so that $f(x, 0)=0$ for a.a. $x \in \Omega$. In this sense we can say that (3.2) is the natural condition to consider in the present context. 


\section{REFERENCES}

1. H. Amann, Liusternik-Schnirelmann theory and nonlinear eigenvalue problems, Math. Ann. 199 (1972), 55-72.

2. M. S. Berger, Nonlinearity and functional analysis (Academic Press, New York, 1977).

3. H. Brezis, Analyse fonctionnelle, theorie et applications (Masson, Paris, 1983).

4. D. G. De Figueiredo, Lectures on the Ekeland variational principle with applications and detours (Tata Inst. of Fundamental Research, Bombay, 1989).

5. J. Dieudonne, Foundations of modern analysis (Academic Press, New York, 1969).

6. M. A. Krasnoselskii, Topological methods in the theory of nonlinear integral equations (Pergamon Press, New York, 1964).

7. G. Prodi and A. Ambrosetti, Analisi non lineare (Scuola Normale Superiore, Pisa, 1973).

8. P. H. Rabinowitz, Variational methods for nonlinear eigenvalue problems, Eigenvalues of nonlinear problems pp. 141-195. (Cremonese, Rome, 1974).

9. M. M. Vainberg, Variational methods for the study of nonlinear operators (Holden-Day, San Francisco, 1964). 1989).

10. J. Mawhin and M. Willem, Critical point theory and Hamiltonian systems (Springer, Berlin,

Dipartimento di MATEMATICA

UNIVERSITÁ dI SiENA

53100 Siena (ITALY)

E-MAIL: chiappinelli@unisi.it 